\title{
Zur Kompatibilität von Maßsystemen der Akustik und zu den Wirkungen von Energie-äquivalenten, zum Gehör jedoch mehr oder weniger kompatiblen Schallbelastungen
}

\author{
Hartmut Irle ${ }^{1} \cdot$ Helmut Strasser ${ }^{1}$ \\ Angenommen: 27. April 2021 / Online publiziert: 31. Mai 2021 \\ (c) Der/die Autor(en) 2021
}

\section{Zusammenfassung}

Die Stärke von Schallbelastungen wird bekanntlich durch den Pegel L mit einem logarithmischen Maß angegeben. Dieses, formal richtig nach dem Weber-Fechner'schen Gesetz aufgebaute Maßstabs-System der Lautstärke in dB (Dezibel) ist allerdings alles andere als empfindungsgerecht und damit inkompatibel zum Gehör. Das zeigt sich z.B. daran, dass ein Pegel von 100 Dezibel (dB) nicht lediglich doppelt so laut empfunden wird wie ein Pegel von 50dB. Ein solcher Messwert repräsentiert sogar das „100.000-fache“ von $50 \mathrm{~dB}$, und damit eine unvorstellbar höhere Belastung. Selbst in einer nur um $3 \mathrm{~dB}$ höheren Belastung steckt bereits die doppelte Energie bzw. Belastung für das Gehör. Weil eben nicht das Weber-Fechner'sche Gesetz, sondern das Stevens'sche Potenz-Gesetz erwiesenermaßen Gültigkeit besitzt, sollte bei der Einstufung der Stärke von Schallbelastungen zumindest das einigermaßen empfindungsgerechte Maßstabs-System der Lautheit mit der Einheit „Sone“ Verwendung finden. Eine Zunahme des Pegels um 10 dB, die erfahrungsgemäß mit einer gefühlten Verdopplung des „Empfindens“ verbunden wird, führt dabei auch zu einer Verdopplung des Sone-Wertes. Das Benutzen des allseits bekannten „dB“ schafft nicht selten Verwirrung und führt oftmals zu gravierenden Fehleinschätzungen. In graphischen Darstellungen werden das inkompatible Maßsystem der Lautstärke und das zum menschlichen Gehör eher kompatible Maßsystem der Lautheit in "Sone“ konkretisiert und veranschaulicht. Dabei werden auch erhebliche Diskrepanzen bei der Beurteilung von Schallexpositionen aufgezeigt, die bei unterschiedlicher Höhe und Einwirkdauer mittels des Halbierungsparameters q=3 als gleich hoch eingeschätzt werden. Auszugsweise dargestellte Ergebnisse aus umfangreichen audiometrischen Untersuchungen belegen, dass energie-äquivalente, aber zeitlich unterschiedlich verteilte Schallbelastungen von in der Arbeit noch zulässigen $85 \mathrm{~dB}(\mathrm{~A}) / 8 \mathrm{~h}$ höchst unterschiedlich starke zeitweilige Hörschellenverschiebungen (Temporary Threshold Shifts, TTS-Werte) verursachen. Ferner wird veranschaulicht, dass auch gleich starke moderne Musikschallbelastungen von $94 \mathrm{~dB} / 1 \mathrm{~h}$ bei otologisch normalen Probanden zu audiometrisch messbaren, ähnlich hohen Hörschwellenverschiebungen wie Industrielärm führen. Das gilt z. B. für „,Heavy Metal“, „Techno“ oder auch „House Music“. Besteht die gleich starke und genauso lang anhaltende akustische Belastung jedoch z. B. aus „Klassischer Musik“, so kommt es allenfalls zu „Physiologischen Kosten“ in der Größenordnung von ca. 1/4 dessen, was zum menschlichen Gehör inkompatible akustische Belastungen verursachen. Daraus ist zu folgern, dass das Gehör nach dem Motto „Der Ton macht die Musik“ mit sinusförmigen akustischen Belastungen eher „klar kommt“ (dazu kompatibel ist) als mit stochastischen und u. U. impulshaltigen Schallereignissen, wie sie durch Metall-auf Metall-Schläge auftreten oder bei Maschinengeräuschen in der Metallverarbeitung vorkommen.

Schlüsselwörter Inkompatibles logarithmisches Maßsystem · Wirkungen akustischer Belastungen · Hörschwellenverschiebungen · Physiologische Kosten des Gehörs.

Dr.-Ing. Hartmut Irle

irle@ergonomie.uni-siegen.de

1 Fachgebiet Arbeitswissenschaft/Ergonomie, Universität Siegen, Paul-Bonatz-Str. 9-11, 57068 Siegen, Deutschland 


\title{
On the compatibility of measurement systems of acoustics and on the effects of energy-equivalent sound exposures that are more or less compatible to the hearing
}

\begin{abstract}
The intensity of noise exposures traditionally is quantified via the level $\mathrm{L}$ as a logarithmic measure. This originally according to Weber-Fechner's law formally correct established system, however, is anything else than in harmony with sensations and, therefore, is not compatible to the human hearing. This may simply be shown via a level of 100 decibel (dB) which is not just twice as high as a level of $50 \mathrm{~dB}$ but represents an energy which is 100,000 times higher, i.e., an unimaginable high exposure. Even a noise level increase of just $3 \mathrm{~dB}$ inheres already a doubling of the energy to the hearing. Since after introducing the logarithmic system, Weber-Fechner's law has proven to be invalid but Steven's power law, indeed, meets much more the psycho-physiological characteristics of the human hearing, the measuring system of loudness with the unit "Sone" should be used. Hereby, an increase of $10 \mathrm{~dB}$ which is generally felt as a doubling of the exposure intensity is represented in a doubling of the "Sone"-value. The particularly common measuring unit "dB" often causes heavy misjudgments of the effects of noise on the hearing. Via graphic illustrations, the incompatible measuring system of the noise intensity (in $\mathrm{dB}$ ) and the rather compatible measuring system of loudness with the unit "Sone" are precisely described and visualized. Also heavy discrepancies were presented when the energy-equivalence principle for varying intensities and noise durations (3-dB exchange rate) is applied as the traditional approach in rating of noise. Epitomized results from comprehensive audiometric research demonstrate that in the working area tolerable energy-equivalent exposures of $85 \mathrm{~dB}(\mathrm{~A}) / 8 \mathrm{~h}$ which vary in intensity and time, cause tremendously varying effects on the hearing which can be quantified in Temporary Threshold Shifts (TTS) in the hearing. It will also be shown that energy-equivalent modern music exposures, such as "Heavy Metal", "Techno" or also "House Music" are associated with TTS-values of otological normal subjects in the same range as a tolerable industrial noise (an exposure of $94 \mathrm{~dB}$ over $1 \mathrm{~h}$, equivalent to a daily noise exposure of $85 \mathrm{~dB} / 8 \mathrm{~h}$ ). For an energetically identical acoustic exposure to "Classical Music", however, the hearing has to pay only approximately $1 / 4$ of the physiological costs associated with industrial noise. That clearly shows that the human hearing follows the slogan "The sound makes the music" or the sentence "The tone is what matters in music". Therefore, it can be concluded that the hearing is much more compatible to sinusoidal sound waves than to stochastic and/or impulse noise stemming from heavy metal strokes or which are caused by metal-workers or by heavy machines.
\end{abstract}

Keywords Incompatible logarithmic measuring system - Effects of acoustic exposures · Hearing threshold shifts . Physiological costs to the hearing

\section{Einführung in die Maßsysteme der Akustik}

Weil die Lärmschwerhörigkeit nach wie vor die am meisten vorkommende Berufskrankheit ist, gilt es im Arbeitsschutz und in der Arbeitswissenschaft, besonderes Augenmerk auf die akustischen Belastungen an Arbeitsplätzen zu richten, und diese zu messen, zu bewerten und zu beurteilen, sowie gegebenenfalls primäre, sekundäre und tertiäre Schallschutzmaßnahmen zu ergreifen.

Im oberen Teil von Abb. 1 ist die formelmäßige Beschreibung der Quantifizierung der Stärke von Schallbelastungen (bekanntlich durch den Pegel L mit einem logarithmischen Maß) dargestellt. Neben dem Schalldruckpegel ist aber, wie im mittleren Teil von Abb. 1 aufgeführt, auch die frequenzmäßige Zusammensetzung der Schallbelastungen zu erfassen. Dabei sollten neben physikalischen Größen wie Oktav- und Terzpegeln $\left(\mathrm{L}_{\mathrm{Oktav}}\right.$ und $\left.\mathrm{L}_{\mathrm{Terz}}\right)$ z. B. mit Phon oder $\mathrm{dB}(\mathrm{A})$ auch physiologisch und psychologisch bewertete Maße gebildet werden. Schließlich spielt auch die Einwirkdauer der Schallbelastungen eine wichtige Rolle. Dazu muss bei zeitlich schwankenden Pegeln nach den im unteren Teil von Abb. 1 aufgeführten Formeln ein Mittelwert $L_{m}$ oder der auf den 8-Stundentag bezogene Beurteilungspegel $\mathrm{L}_{\mathrm{r}}$ bzw. nach der Lärm-Vibrations-Arbeitsschutzverordnung (N. N. 2007) der Tages-Lärmexpositionspegel gebildet werden.

Dieses, formal richtig nach dem Weber-Fechner'schen Gesetz aufgebaute Maßstabssystem der Lautstärke in dB (Dezibel), wonach absolute Empfindungsänderung das Ergebnis relativer Reizänderungen sein sollten (vgl. oberen Teil von Abb. 2), ist allerdings alles andere als empfindungsgerecht und damit inkompatibel zum Gehör. Das zeigt sich z.B. daran, dass ein Pegel von 100 Dezibel (dB) nicht lediglich doppelt so laut empfunden wird wie ein Pegel von $50 \mathrm{~dB}$. Ein solcher Messwert repräsentiert sogar das „100.000-fache“ von $50 \mathrm{~dB}$, und damit eine unvorstellbar höhere Belastung. Selbst in einer nur um $3 \mathrm{~dB}$ höheren Belastung steckt bereits die doppelte Energie bzw. Belastung für das Gehör. Es muss aber eine akustische Belastung um 


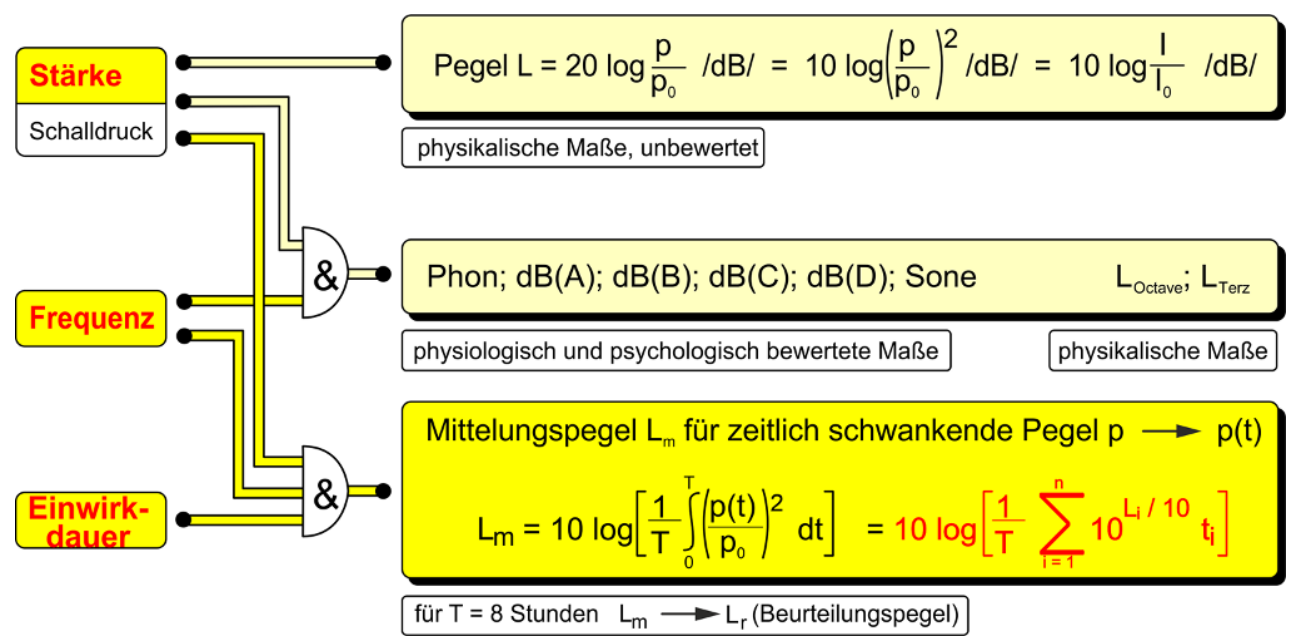

Abb. 1 Kennwerte des Schalls und Zusammenfassung seiner 3 Dimensionen Stärke, Frequenz und Einwirkdauer zu einem zeitlichen Mittelwert $\mathrm{L}_{\mathrm{m}}$ bzw. zu dem, auf den 8-Stunden-Tag bezogenen Tages-Lärmexpositionspegel (bzw. Beurteilungspegel $\mathrm{L}_{\mathrm{r}}$ ). (Aus Strasser 2011)

Fig. 1 Characteristics of sound exposure measures and gradual assessment of the 3 physical dimensions intensity, frequency and exposure time, leading to a time-averaged value $\mathrm{L}_{\mathrm{m}}$ and the rating level $\mathrm{L}_{\mathrm{r}}$, averaged over an 8-hour-working day ("Beurteilungspegel", meanwhile named "TagesLärmexpositionspegel”), respectively. (Source: Strasser 2011)

Abb. 2 Inkompatibles Maßstabssystem der Lautstärke und empfindungsgerechte Maßeinheit der Lautheit in „Sone“. (Aus Strasser 2011)

Fig. 2 Incompatible logarithmic scale of sound pressure level in $\mathrm{dB}$ and compatible scale of loudness with linear units in "Sone" due to sensation. (Source: Strasser 2011)
Inkompatibles logarithmisches Maßstabssystem der Lautstärke in dB

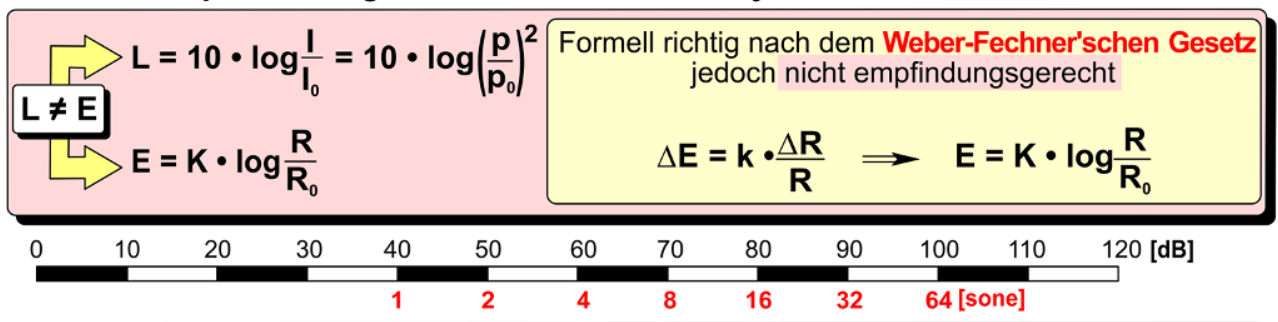

\begin{tabular}{|c|c|}
\hline$\Rightarrow S=2^{(L-40) / 10}$ & $\begin{array}{l}\text { Formell richtig und empfindungsgerecht } \\
\text { nach dem Stevens'schen Potenzgesetz }\end{array}$ \\
\hline$\underbrace{S=E} E=2^{(L-40) / 10}$ & $\frac{\Delta E}{E}=k \cdot \frac{\Delta R}{R} \Rightarrow E=E_{0} \cdot\left(\frac{R}{R_{0}}\right)^{k}=K^{\prime} \cdot\left(\frac{I}{I_{0}}\right)^{K}=2^{(L-40) / 10}$ \\
\hline
\end{tabular}

Kompatibles, empfindungsgerechtes Maßstabssystem der Lautheit in Sone

\begin{tabular}{|ll|}
\hline $\mathrm{R}$ & Physikalischer Reiz \\
$\mathrm{R}_{0}$ & Schwellenreiz \\
$\Delta \mathrm{R}$ & Reizänderung \\
$\mathrm{E}$ & Subjektive Empfindung \\
$\mathrm{E}_{0}$ & Empfindungsschwelle \\
$\Delta \mathrm{E}$ & Empfindungsänderung \\
$\mathrm{k}, \mathrm{K}, \mathrm{K}^{\prime}$ & Konstante \\
\hline
\end{tabular}

I Schallintensität

$\mathrm{I}_{0}$ Schallintensität an der Hörschwelle bei $1000 \mathrm{~Hz}\left(2 \cdot 10^{-12} \mathrm{~W} / \mathrm{m}^{2}\right)$

p Schalldruck

$\mathrm{p}_{0}$ Schalldruck an der Hörschwelle bei $1000 \mathrm{~Hz}\left(2 \cdot 10^{-5} \mathrm{~Pa}\right)$

L Pegel als Messgröße für die Lautstärke

S Empfindungsgerechte Messgröße für die Lautheit
$10 \mathrm{~dB}$ höher sein, dass sie auch doppelt so laut empfunden wird.

Weil eben nicht das Weber-Fechner'sche Gesetz, sondern das Stevens'sche Potenzgesetz erwiesenermaßen Gültigkeit besitzt, sollte bei der Einstufung der Stärke von Schallbelastungen zumindest das einigermaßen empfindungsgerechte Maßstabssystem der Lautheit mit der Einheit „Sone“ Verwendung finden. Wie in Abb. 2 Mitte dargestellt, sind aus den $\mathrm{dB}$-Werten nach der Formel $\mathrm{S}=2^{(\mathrm{L}-40) / 10}$ Sone-Werte $\mathrm{zu}$ berechnen. Die Formel baut auf dem Stevens'schen Potenz- gesetz auf, wonach nicht absolute, sondern relative Empfindungsänderungen das Resultat von relativen Reizänderungen sind. Diese empfindungsgerechte Maßeinheit der Lautheit beginnt bei 1 Sone $(40 \mathrm{~dB})$. Ein Pegel von $10 \mathrm{~dB}$ mehr - also $50 \mathrm{~dB}$ - entsprechen dem Wert von 2 Sone. Schließlich führt jede weitere Erhöhung um $10 \mathrm{~dB}$ zu einer Verdopplung des Sone-Wertes $(50 \mathrm{~dB}=2$ Sone, $60 \mathrm{~dB}=4$ Sone und z.B. $100 \mathrm{~dB}=64$ Sone). Bezüglich Details und weiterer kompatibler psycho-akustischer Maßsysteme sei auf die 


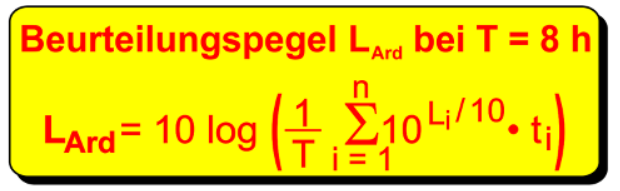

\section{$L[\mathrm{~dB}(\mathrm{~A})]$}

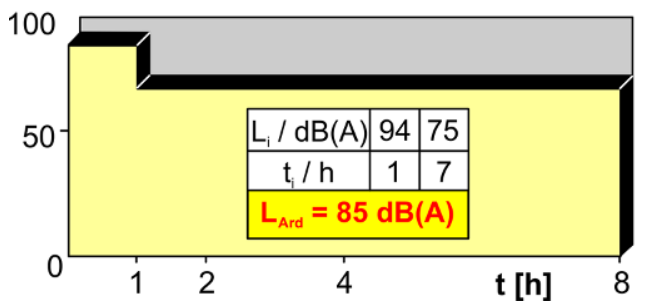

$L[\mathrm{~dB}(\mathrm{~A})]$

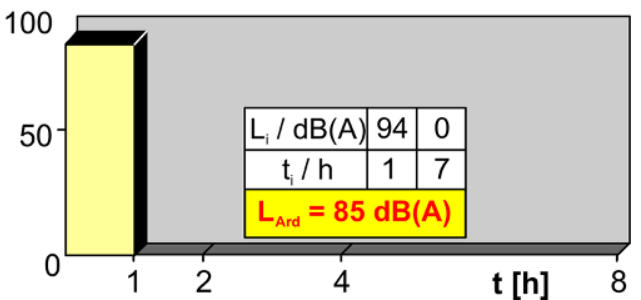

Abb. 3 Beispiel für das zulässige Auffüllen von Ruhephasen mit Lärm ohne Auswirkungen auf den Tages-Lärmexpositionspegel bzw. den Beurteilungspegel $\mathrm{L}_{\text {Ard, }}$ wenn der zusätzliche Lärm (in diesem Fall $75 \mathrm{~dB}$ über $7 \mathrm{~h}$ ) um ca. $20 \mathrm{~dB}$ unter dem dominierenden Spitzenpegel von $94 \mathrm{~dB} / 1 \mathrm{~h}$ liegt. (Aus Strasser 2011)

Fig. 3 Example for the allowable filling-up of resting periods with noise without any effect on the rating level LArd ("Beurteilungspegel" and "Tages-Lärmexpositionspegel", respectively) when the additional noise (in this case $75 \mathrm{~dB}$ during $7 \mathrm{~h}$ ) remains around $20 \mathrm{~dB}$ below the peak level of $94 \mathrm{~dB}$ for $1 \mathrm{~h}$. (Source: Strasser 2011)

letzte Ausgabe eines „Klassikers der Akustik“, herausgegeben von Fastl und Zwicker (2007), verwiesen.

Das Benutzen des allseits bekannten „dB“ schafft nicht selten Verwirrung und führt oftmals, ebenso wie die alleinige Verwendung des A-Filters in allen Dynamikbereichen, zu gravierenden Fehleinschätzungen. Gleiches gilt, wenn nach der Lärm- und Vibrations-Arbeitsschutzverordnung (N. N. 2007) eine 8-stündige akustische Belastung mit einem Einzahlwert, dem Tages-Lärmexpositionspegel (früher besser bekannt als Beurteilungspegel $\mathrm{L}_{\mathrm{r}, \text {, }}$ (rating Level)) ausgewiesen wird. Die Berechnung von Tages-Lärm- expositionspegeln (Beurteilungspegeln) führt manchmal zu geradezu „verrückten“ bzw. irreführenden Ergebnissen. So ergibt sich nach dem Halbierungsparameter $\mathrm{q}=3$ bei Halbierung bzw. Verdopplung der Expositionszeit ein um $3 \mathrm{~dB}$ höherer bzw. niedriger Wert. So entspricht z.B., wie im rechten Teil von Abb. 3 visualisiert, ein Pegel von $94 \mathrm{~dB}$, der $1 \mathrm{~h}$ einwirkt, einem Beurteilungspegel $\mathrm{L}_{\mathrm{Ard}}$ von $85 \mathrm{~dB}$ über $8 \mathrm{~h}$. In den übrigen $7 \mathrm{~h}$ könnte aber auch (wie im linken Teil von Abb. 3 dargestellt) ein Pegel bis zu $70 \mathrm{~dB}$ vorliegen, ohne dass sich der Beurteilungspegel zahlenmäßig überhaupt ändert. Man könnte also sogar Ruhephasen mit Lärm bis zu
Abb. 4 Energie-äquivalente Pegel-Wirkzeit-Konstellationen mit gleichem Beurteilungspegel $\mathrm{L}_{\mathrm{Ard}}=85 \mathrm{~dB}(\mathrm{~A})$ bei Anwendung des Halbierungsparameters $q=3$, d. h. formelmäßig korrekte, letztlich aber inkompatible Equilibrierung von Dauer- und Impulsschall. (Aus Strasser 2011)

Fig. 4 Energy-equivalent constellations of sound pressure levels and durations ending up in an equal rating level ("Beurteilungspegel") $\mathrm{L}_{\text {Ard }}$ of $85 \mathrm{~dB}(\mathrm{~A})$ when applying the $3 \mathrm{~dB}$-exchange rate-a formally correct procedure but finally leading to an incompatible equilibration of continuous and impulse noise. (Source: Strasser 2011)

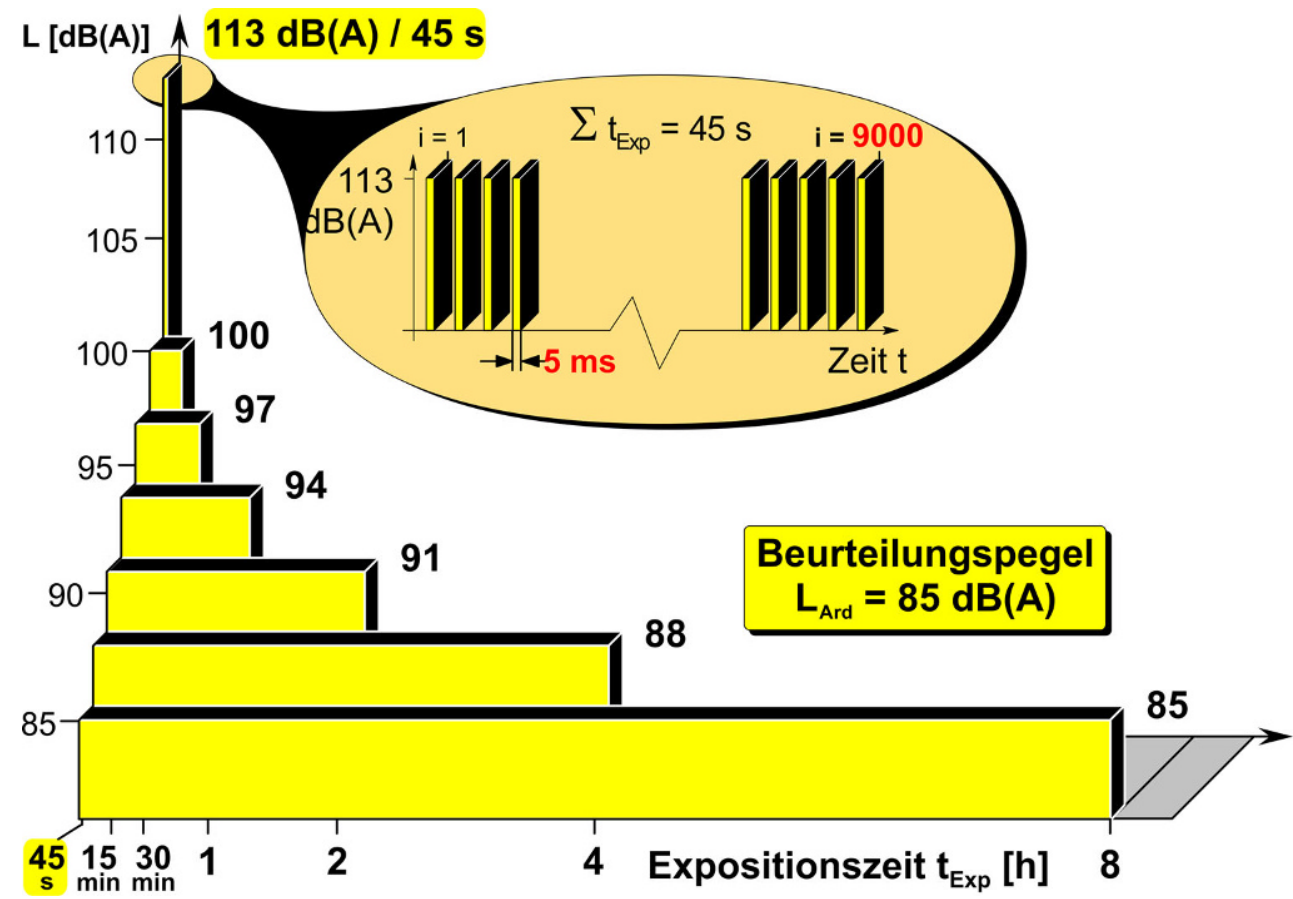


diesem Wert auffüllen, ohne dass das zahlenmäßig im Beurteilungspegel erkennbar wird, wenngleich die Wirkungen auf den Menschen höchst unterschiedlich sind. Andererseits würden Schallschutzmaßnahmen an Lärmquellen, die den Pegel von $75 \mathrm{~dB}$, der über $7 \mathrm{~h}$ einwirkt, vollkommen eliminieren, ,,ad absurdum“ geführt, weil der Tages-Lärmexpositionspegel sich überhaupt nicht ändern würde.

\section{Beispiele für höchst unterschiedliche Wirkungen Energie-äquivalenter Schallbelastungen}

Wie in Abb. 4 visualisiert, entsprechen also bei Anwendung des Halbierungsparameters $\mathrm{q}=385 \mathrm{~dB} / 8 \mathrm{~h}$ einem um $3 \mathrm{~dB}$ höheren Pegel, also $88 \mathrm{~dB} / 4 \mathrm{~h}, 91 \mathrm{~dB} / 2 \mathrm{~h}, 94 \mathrm{~dB} / 1 \mathrm{~h}$, $97 \mathrm{~dB} / 1 / 2 \mathrm{~h}, 100 \mathrm{~dB} / 1 / 4 \mathrm{~h}$ oder auch $113 \mathrm{~dB} / 45 \mathrm{~s}$. Schließlich wird selbst das „Aufsplitten“ dieser kurzen akustischen Belastung in Impulsschall (z. B. 9000 Hammerschläge auf einen Amboss mit einer Dauer von jeweils $5 \mathrm{~ms}$ ) als unbedenklich deklariert.

Zur Problematik bei der derzeitigen Ermittlung des Beurteilungspegels nach der DIN 45645-2 (2012), die spezi- ell für Geräuschbelastungen geschaffen wurde, bei denen lediglich von extra-auralen Wirkungen und nicht von gehörschädigendem Lärm auszugehen ist, sei auf das Positionspapier von Strasser (2017) verwiesen. Leider sind auch derartige Normen und Richtlinien wie bspw. die VDI 2058 (2014) überhaupt nicht kompatibel zu den Wirkungen, weil nicht davon ausgegangen werden kann, dass die Gleichung „Energie-Äquivalenz = Stör-Äquivalenz“ gilt. Oder darf etwa ein einzelnes Schallereignis mit $90 \mathrm{~dB}(\mathrm{~A})$ (als Vorbeifahrtpegel eines Lastkraftwagens) mit 1000 Schallereignissen von jeweils ca. $60 \mathrm{~dB}(\mathrm{~A})$ (herrührend von Personenkraftwagen) gleichgesetzt werden? Der LKW stört dabei (vielleicht etwas stärker) nur einmal für ein paar Sekunden, wohingegen die 1000 PKWs über den ganzen Tag verteilt, eine äußerst lästige Schallquelle darstellen können.

Laute Schallbelastungen bzw. Lärm mit einem Beurteilungspegel oberhalb $85 \mathrm{~dB}(\mathrm{~A})$ führen bekanntlich $\mathrm{zu}$ drastischen Vertäubungen, mitunter sogar zu Schädigungen des Gehörs. Selbst wenn Hörschwellenanhebungen (Vertäubungen) nur $10 \mathrm{~dB}$ betragen, wird der Höreindruck bereits halbiert. Nicht selten vorkommende Hörschwellenverschiebungen in der Größenordnung um $20 \mathrm{~dB}$, die oft noch

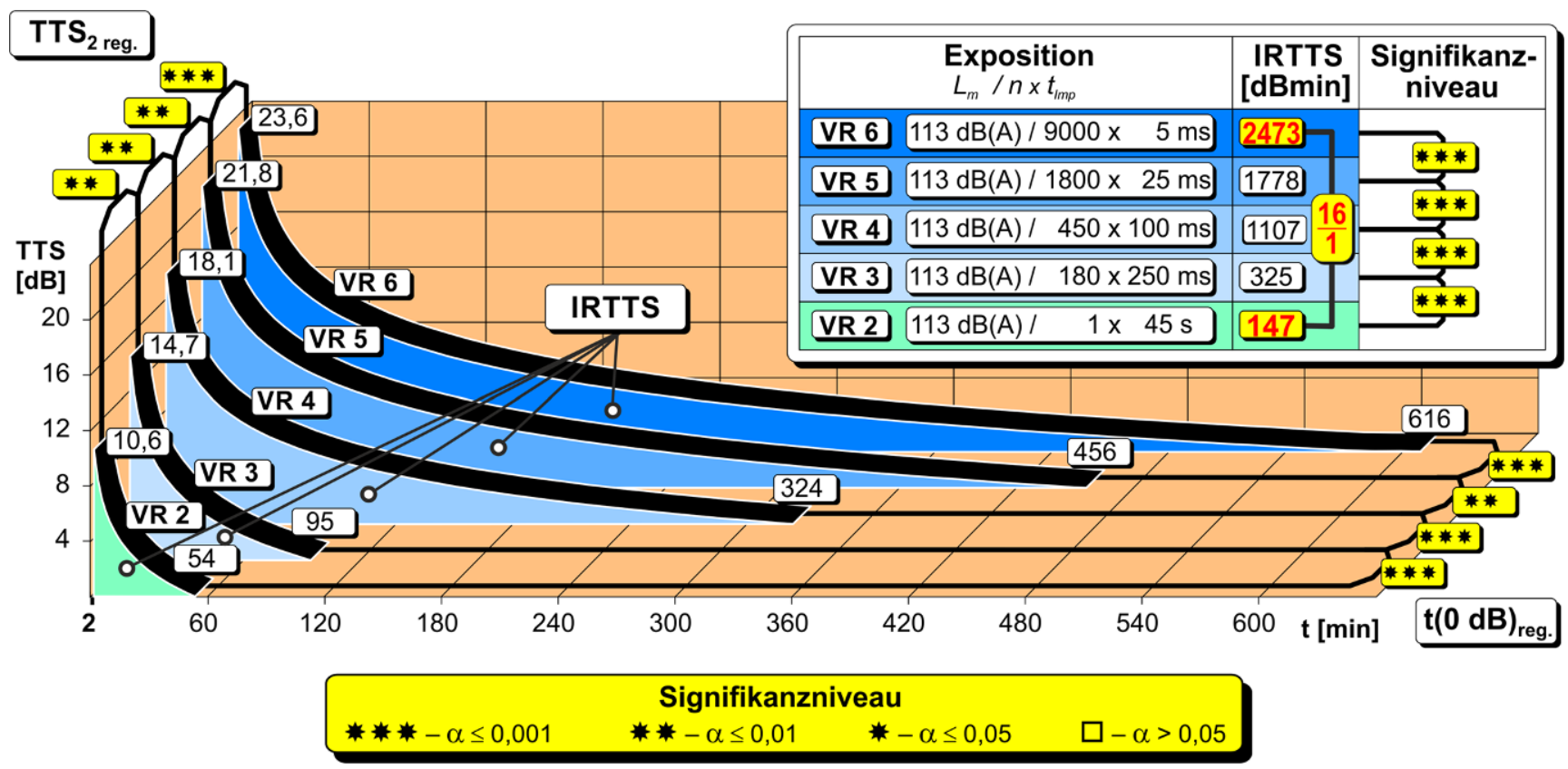

Abb. 5 Restitutionsverläufe $T T S(t)$, d.h. physiologische Reaktionen des Gehörs auf verschiedene Expositionen mit gleichem Expositionspegel $\mathrm{L}_{\mathrm{m}}=113 \mathrm{~dB}(\mathrm{~A})$ und gleicher Expositionszeit texp $\left(n \times \mathrm{t}\right.$ Imp) in den Versuchsreihen (VR 2-VR6) mit den Kennwerten $T T S_{2}$ reg, $t(0 d B)$ reg und den aufsummierten physiologischen Kosten IRTTS, die das Gehör für die energetisch gleichen Belastungen (alle äquivalent zu $85 \mathrm{~dB}(\mathrm{~A}) / 8 \mathrm{~h}$ bzw. $94 \mathrm{~dB}(\mathrm{~A}) / 1 \mathrm{~h}$ ) zu ,bezahlen“ hatte, d. h. Auswirkungen des „Aufsplittens“ von Dauerschall in Impulsschall mit unterschiedlich langen Einzelimpulsen. (Symbolische Kennzeichnung der Signifikanz von Unterschieden zwischen den „Antworten“ des Gehörs auf die verschiedenen Belastungen gemäß des 1-seitigen WILCOXON-Tests). (Quelle: Irle et al. 2006)

Fig. 5 Restitution time courses $\operatorname{TTS}(t)$, i.e., physiological responses of the hearing to various expositions with an identical exposure level $\mathrm{L}_{\mathrm{m}}=113 \mathrm{~dB}(\mathrm{~A})$ and exposure time $\mathrm{t}_{\mathrm{Exp}}\left(n \times \mathrm{t}_{\mathrm{Imp}}\right)$ in the test series VR 2-VR 6, with characteristics $T_{T S} S_{2 e g}, t(0 \mathrm{~dB})$ reg and total physiological cost IRTTS the hearing has to pay for energy-equivalent exposures (all equivalent to $85 \mathrm{~dB}(\mathrm{~A}) / 8 \mathrm{~h}$ or $94 \mathrm{~dB}(\mathrm{~A}) / 1 \mathrm{~h}$ ) revealing the effects of splittingup continuous noise into shorter and shorter impulses. (Symbolic labeling of significance levels for the differences between the responses to the various exposures according to the 1-tailed WILCOXON-test). (Source: Irle et al. 2006) 
Stunden nach einer Schallbelastung anhalten, reduzieren den Lautheitseindruck auf $1 / 4$.

Experimentelle Untersuchungen aus den 70er-Jahren des letzten Jahrhunderts z. B. von Miller (1974) und umfangreiche experimentelle Messungen an größeren Gruppen von otologisch unauffälligen Testpersonen (Details siehe Irle et al. 1998, 2006) haben ergeben, dass der Halbierungsparameter nur bei akustischen Belastungen im Stundenbereich angewandt werden darf, und dass bei sehr kurzdauernden, zu $85 \mathrm{~dB} / 8 \mathrm{~h}$ energie-äquivalenten Belastungen von z.B. $113 \mathrm{~dB} / 45 \mathrm{~s}$ kaum noch Vertäubungen auftreten. Es konnte aber experimentell von Hesse et al. (1994) belegt werden, dass bei der Aufsplittung von derartigen Schallbelastungen in Impulsschall erheblich höhere und lang anhaltende Vertäubungen auftreten (vgl. Abb. 5 und rechte Seite von Abb. 6). So ist z. B. das Gehörschadens-Risiko für typischen Impulsschall (9000 Einzelimpulse mit $113 \mathrm{~dB}(\mathrm{~A})$ einer Dauer von jeweils $5 \mathrm{~ms}$ ) ca. 2,5-mal so hoch wie von Dauerschall mit $94 \mathrm{~dB}(\mathrm{~A})$ über $1 \mathrm{~h}$. Bei einer energie-äquivalenten Belastung von $113 \mathrm{~dB}(\mathrm{~A}) /$ über $45 \mathrm{~s}$ liegt es dagegen bei nur $15 \%$.

Zur Quantifizierung von Hörschwellenverschiebungen und deren Restitution bzw. den „Physiologischen Kosten“, die das Gehör für vorausgehende, verschiedenartige akustische Belastungen zu bezahlen hat, siehe z.B. Irle und Strasser (2005), Irle et al. (2006) oder Strasser und Irle (2006) sowie Strasser et al. (2007, 2008a). Die Anwendung des Halbierungsparameters für extrem hohe Pegel und sehr kurze Wirkzeiten ist absolut unangemessen, weil bei Überschreiten von $120 \mathrm{~dB}$ nicht nur mit Vertäubungen, d.h. einer reversiblen Ermüdung des Gehörs, sondern mit akuten irreversiblen Schädigungen gerechnet werden muss.
Experimentelle Untersuchungen in diesem Dynamikbereich an Menschen sind aus ethischen Gründen verboten.

Energetisch gleiche Musikschallbelastungen können - wie z. B. von Irle et al. (2005), Strasser et al. (2005) oder Strasser et al. (2008b) nachgewiesen - das Gehör ähnlich stark in Mitleidenschaft ziehen wie Industrielärm. Das gilt z. B. für „Heavy Metal“, „Techno“ oder auch „House Music". Besteht die gleich starke und genauso lang anhaltende akustische Belastung jedoch z. B. aus „Klassischer Musik“ oder auch „Klassischer" Chinesischer Musik (Details siehe Strasser et al. 2009), so kommt es allenfalls zu „Physiologischen Kosten“, die das Gehör in Form von Vertäubungen zu bezahlen hat, in der Größenordnung von höchstens 1/4 dessen, was zum menschlichen Gehör inkompatible SchallBelastungen verursachen. Details hierzu siehe Abb. 7, 8 und 9.

Daraus ist eindeutig zu folgern, dass das Gehör nach dem Motto „Der Ton macht die Musik“ mit sinusförmigen akustischen Belastungen eher „klar kommt“ (dazu also kompatibel ist) als mit stochastischen und u.U. impulshaltigen Schallereignissen, wie sie durch Metall-auf Metall-Schläge auftreten oder die bei Maschinengeräuschen in der Metallverarbeitung vorkommen.

Bei der traditionellen Beurteilung von Schallbelastungen werden in der Regel auch keine Pegel-Zeit-Analysen vorgenommen oder Pegel-Verteilungen ermittelt, wobei aus experimentellen Untersuchungen mittlerweile bekannt ist, dass z. B. bei „Klassischer Musik“ mit immer wiederkehrenden ruhigen Passagen nach lauteren Phasen (Decrescendos nach Crescendos) das Gehör die Gelegenheit bekommt, zwischendurch immer mal wieder „Durchzuschnaufen“ bzw. zu „Atmen“, anders als z.B. bei der Dauerbeschallung bzw. geradezu einem „Trommelfeuer“
Abb. 6 Gehörschadens-Risiko-Wert energie-äquivalenter Dauer- und Impulsschall-Expositionen, bestimmt über den Quotienten aus den IRTTS-

Werten der Versuchsreihen VR 2-VR 6 und dem IRTTS-Wert, der sich aus der Exposition mit $94 \mathrm{~dB}(\mathrm{~A}) / 1 \mathrm{~h}$ (VR 1 als Referenzwert $=1,00)$ ergeben hatte. (Quelle: Hesse et al. 1994)

Fig. 6 Hearing damage-risk criterion of energy-equivalent continuous and impulse noise exposures, determined via relating the IRTTS-values of the test series VR 2-VR 6 to the reference IRTTS-value associated with $94 \mathrm{~dB}(\mathrm{~A}) / 1 \mathrm{~h}$ (VR 1) (reference value $=1.00)$. (Source: Hesse et al. 1994)

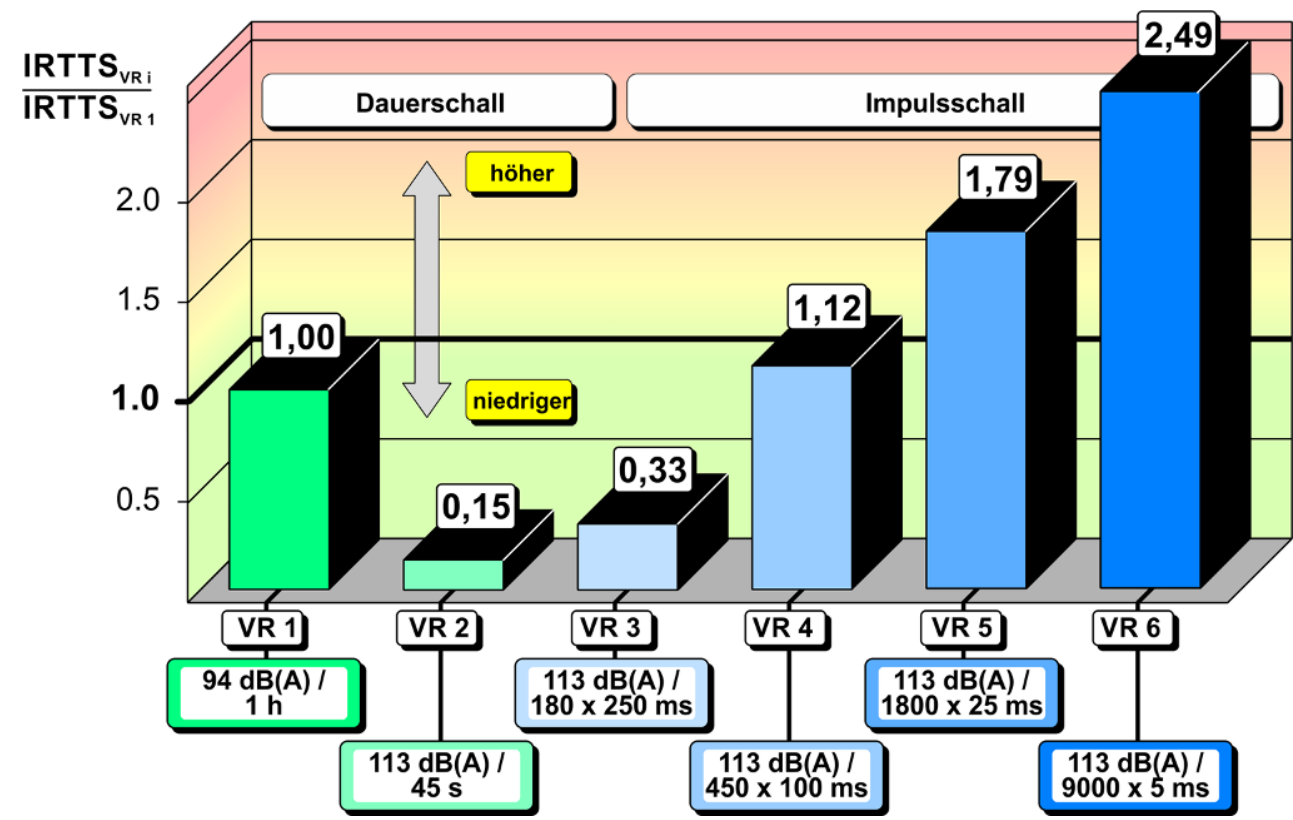




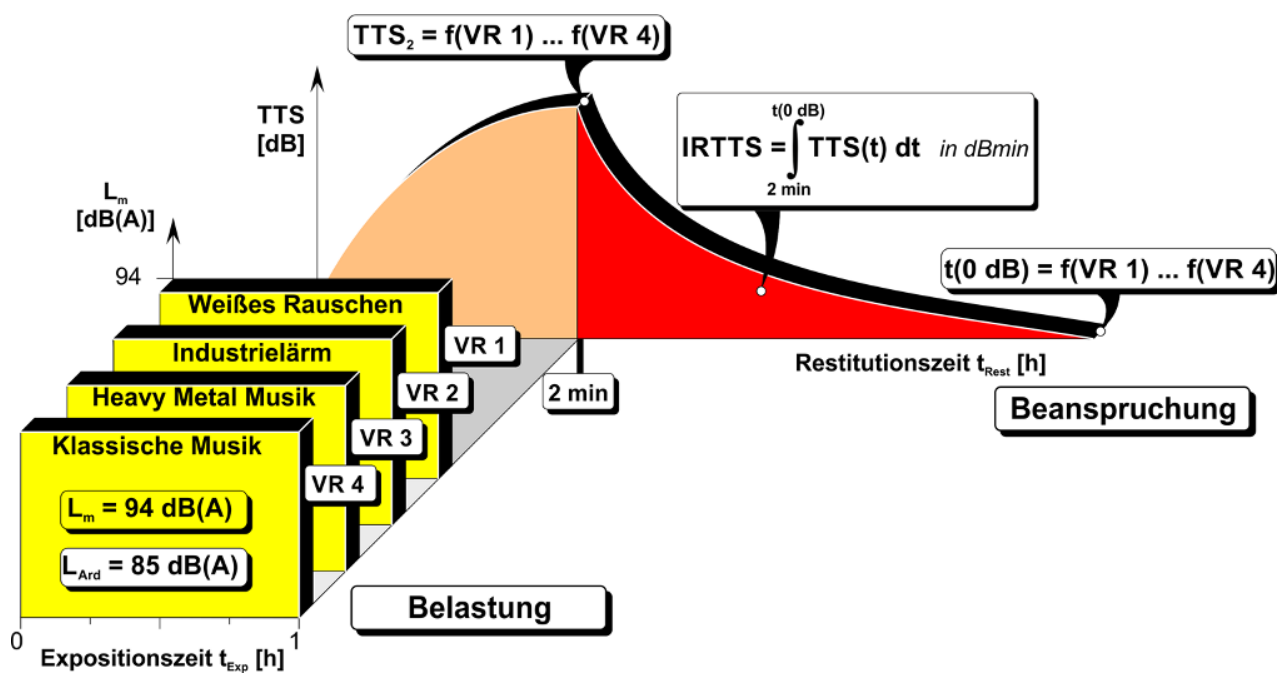

Abb. 7 Schematische Darstellung der energie-äquivalenten Expositionen „Weißes Rauschen“ (einer akustischen Referenzbelastung mit gleicher Energie in allen Frequenzbereichen), „Industrielärm“, „Heavy Metal Musik“, und „Klassische Musik“ (Belastung jeweils 94 dB/1 h) und hypothetisch erwartete „Physiologische Antwort“ ( $\mathrm{TTS}_{2}$, Restitutionszeit $\mathrm{t}(0 \mathrm{~dB})$ und Gesamtsumme der Vertäubungen, d.h. Integrated Restitution Temporary Threshold Shifts IRTTS). (Quelle: Strasser et al. 2005)

Fig. 7 Schematic representation of the energy-equivalent exposures "White Noise" (an acoustic reference exposure with equal energy in all frequency areas), "Industrial Noise", "Heavy Metal Music", and "Classical Music" (94 dB(A)/1 h, each), and hypothetically expected "Physiological Responses" (TTS 2 , Restitution Time $\mathrm{t}(0 \mathrm{~dB})$ and Integrated Restitution Temporary Threshold Shift IRTTS). (Source: Strasser et al. 2005)

Abb. 8 Energie-äquivalente, gleich laute und gleich lange Lärm- und Musikschall-Expositionen (jeweils $94 \mathrm{~dB}(\mathrm{~A}) / 1 \mathrm{~h})$. (Quelle: Strasser et al. 2005)

Fig. 8 Energy-equivalent sound and music exposures with a mean level of $94 \mathrm{~dB}$ and a duration of $1 \mathrm{~h}$, each. (Source: Strasser et al. 2005)
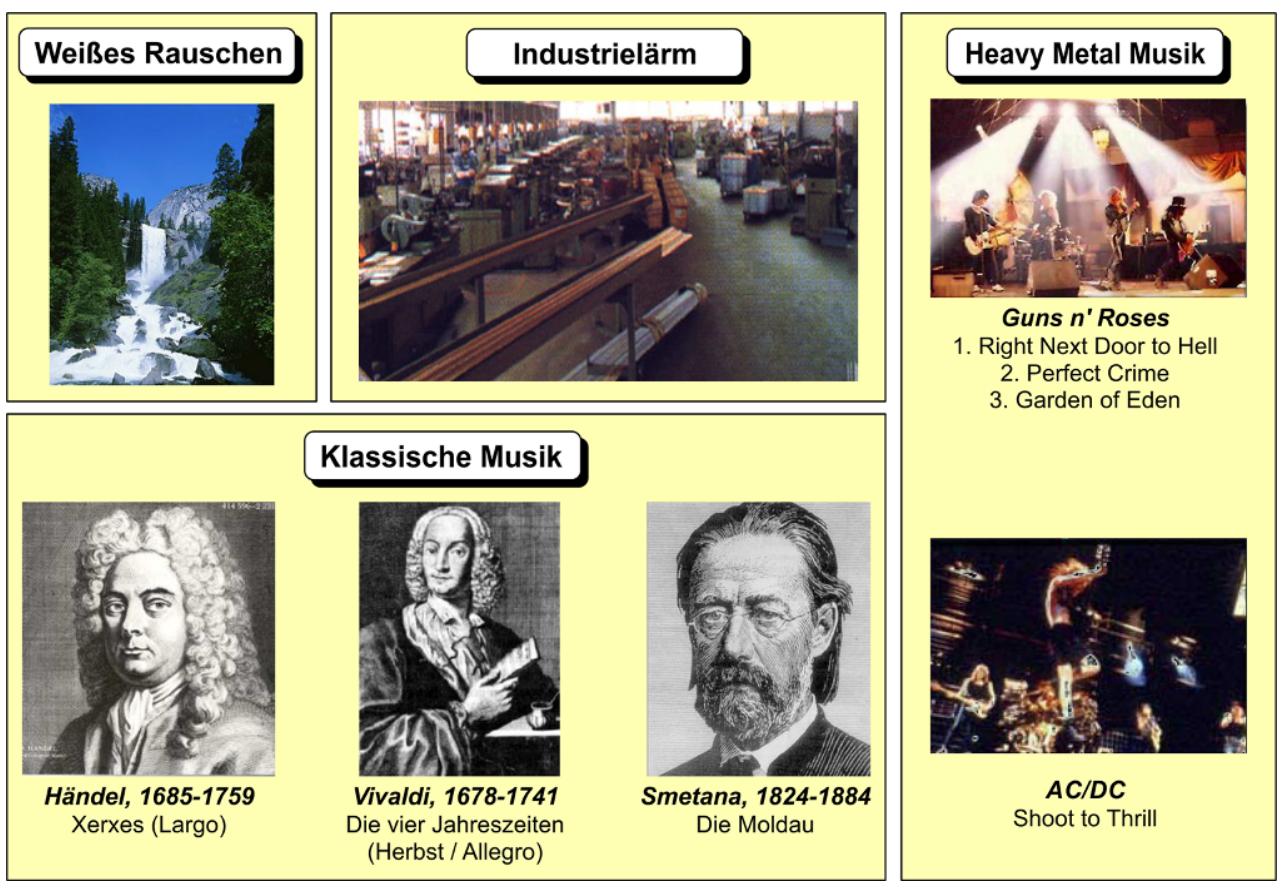

mit 120 „Schlägen“/min bei „House Music“, wobei sogar bewusst durch hohe Anteile im tieferen Frequenzbereich Resonanzen im ganzen Körper verursacht werden. Das ist durchaus allgemein erkennbar, wenn z.B. ein PKW beim Warten an einer roten Ampel und entsprechender moderner Musik, die sich der Lenker „gönnt“, geradezu vibriert. Natürlich ist derartige Musik keineswegs kompatibel zum menschlichen Gehör, das „natürliche“ Geräusche wie z.B. das „Rauschen“ eines Wasserfalls (ähnlich zu „Weißem Rauschen") und sinushaltige Schallvorgänge deutlich besser ,,verträgt“.

Die besondere Gefährlichkeit moderner Musikproduktionen, wie „Heavy Metal“, „Hardrock“ oder „Techno“ liegt darüber hinaus auch noch darin, dass sie in Diskotheken, Open-Air-Konzerten oder über Walkman bei wesentlich höheren Lautstärken gehört werden als Kompositionen, die bei 


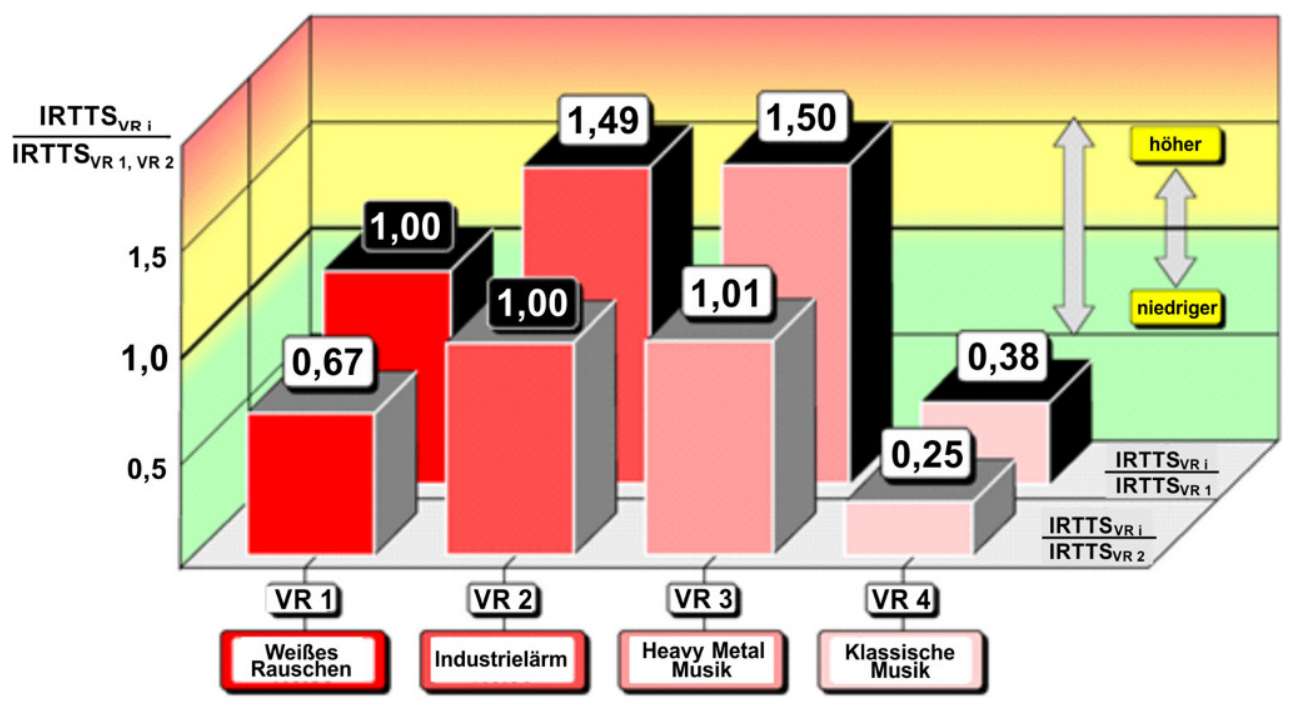

Abb. 9 Relative „Physiologische Kosten“ der verschiedenen energie-äquivalenten Expositionen (in VR 1, VR 2, VR 3 und VR 4), bezogen auf die IRTTS-Werte von VR 2 „Industrielärm“ und VR 1 „Weißes Rauschen“. Zahlen größer 1,00 repräsentieren erhöhte und Zahlen kleiner 1,00 verdeutlichen reduzierte „Physiologischen Kosten“

Fig. 9 Relative "Physiological Costs" of the various energy-equivalent exposures (in VR 1, VR 2, VR 3 und VR 4), quantified by using the IRTTSvalues of "Industrial Noise" (in VR 2) or "White Noise" (in VR 1) as a reference. Numbers above 1 indicate increased, numbers smaller than 1.00 indicate reduced "Physiological Costs"

Symphoniekonzerten oder Opernaufführungen dem Publikum dargeboten werden.

\section{Diskussion und Ausblick}

Bei der konventionellen Beurteilung von Umgebungsbelastungen (also nicht nur von Schallbelastungen) werden - auf den ersten Blick zwar plausibel - in der Regel hohe Intensitäten bei kurzen Wirkzeiten gleich eingeschätzt wie niedrige Intensitäten bei entsprechend längeren Expositionen. Dieses Prinzip einer energie-äquivalenten Verrechnung basiert auf der Hypothese, dass eine gleiche Dosis auch gleiche Wirkungen habe. Das ist jedoch keineswegs konform mit Paracelsus, auf den sich die Vertreter der Dosis-Maxime bzw. des Energie-Äquivalenz-Prinzips gerne beziehen. Solche Denkweisen sind mehr an physikalischen Paradigmen orientiert als an den psycho-physiologischen Eigengesetzlichkeiten des Menschen. Wer genau bei Paracelsus nachschlägt - dem der Spruch „Dosis facit venenum“ zugeschrieben wird - wird feststellen, dass sich in dem Spruch die Erfahrung und Erkenntnis des Arztes widerspiegelt, dass ein Medikament (der Extrakt einer Heilpflanze) in mehreren Gaben (das ,rechte Maß“) heilbringend ist, wogegen die gesamte Menge auf einmal (die Dosis) tödlich sein kann. Das Gehör „funktioniert" keinesfalls wie ein Dosimeter, das die über den Tag verteilte Schallenergie akkumuliert, um sie dann mathematischen Verrechnungen nach der Dosis-Maxime unterwerfen zu können.
Das gilt auch für andere Sinnesorgane. Ansonsten wären ja auch lokale Temperaturerhöhungen von noch so kurzer Dauer, z.B. beim Berühren der Fingerkuppe einer heißen Herdplatte, locker in eine momentane wohlige Wärme des Gesamtorganismus umrechenbar, würde ein Lichtblitz oder ein Laserstrahl, der die Netzhaut trifft, nicht blenden oder sogar Schaden anrichten, würde ein kurzer Nadelstich nicht Schmerz hervorrufen, sondern als taktile Streicheleinheit der betroffenen Stelle über eine längere Zeitspanne interpretiert werden dürfen.

Ohne grundsätzliches Wissen um die Kompatibilität von physikalischen Reizen und den physiologischen und psychologischen Eigengesetzlichkeiten des Menschen, d.h. den Reaktionen der Sinnesorgane kommt es im Falle von Schallbelastungen zu geradezu ,unsäglichen“ Gleichsetzungen von gesetzlich sogar noch zulässigen Knallbelastungen (z.B. 100 Ereignisse mit einem Pegel von $140 \mathrm{~dB}$ bei einer Dauer von jeweils $1 \mathrm{~ms}$ ) oder einem (erfreulicherweise gesetzlich nicht mehr ohne Gehörschutz zulässigen) Gewehrschuss, der bei einem Pegel von $160 \mathrm{~dB}$ lediglich $1 \mathrm{~ms}$ dauert. Bei letzterem würde man eine „Ohrfeige“ geradezu in Streicheleinheiten umrechnen.

In Anbetracht der in diesem Beitrag nur auszugsweise darstellbaren Erkenntnisse aus vielfältigen audiometrischen Untersuchungen zu den auralen Wirkungen verschiedenster akustischer, auch schmal- oder breitbandiger, hoch- oder tief-frequenter Schallbelastungen (Zusammenstellung siehe Strasser 2005) wäre es unverantwortlich, sich im Arbeitsschutz und in der Ergonomie auf die bequeme Position einer lediglich physikalischen Betrachtungsweise von Belastun- 
gen zu beschränken, und dabei zu ignorieren, dass Menschen auf Expositionen nach physiologischen und psychologischen Eigengesetzlichkeiten reagieren, und eben nicht lediglich nach der Physik der leblosen Materie ,funktionieren“. Die konventionelle, in Normen und Richtlinien nahegelegte Vorgehensweise bei der Messung, Bewertung und Beurteilung von Schallbelastungen ist zwar hochpräzise und mathematisch exakt. Aus ergonomischer Sicht ist sie jedoch als sehr problematisch einzuschätzen, weil bei der Etablierung von Mess- und Beurteilungsgrößen keineswegs an Kompatibilität mit den Wirkungen auf den Menschen gedacht wurde. Der „Gipfel“ an Unverständlichkeit des logarithmisch geprägten Maßsystems wird „,abgeschossen“ mit dem Titel des von Hoffmann und v. Lüpke (1979) herausgegebenen Buches: „Null Dezibel + Null Dezibel= Drei Dezibel“.

Funding Open Access funding enabled and organized by Projekt DEAL.

Open Access Dieser Artikel wird unter der Creative Commons Namensnennung 4.0 International Lizenz veröffentlicht, welche die Nutzung, Vervielfältigung, Bearbeitung, Verbreitung und Wiedergabe in jeglichem Medium und Format erlaubt, sofern Sie den/die ursprünglichen Autor(en) und die Quelle ordnungsgemäß nennen, einen Link zur Creative Commons Lizenz beifügen und angeben, ob Änderungen vorgenommen wurden.

Die in diesem Artikel enthaltenen Bilder und sonstiges Drittmaterial unterliegen ebenfalls der genannten Creative Commons Lizenz, sofern sich aus der Abbildungslegende nichts anderes ergibt. Sofern das betreffende Material nicht unter der genannten Creative Commons Lizenz steht und die betreffende Handlung nicht nach gesetzlichen Vorschriften erlaubt ist, ist für die oben aufgeführten Weiterverwendungen des Materials die Einwilligung des jeweiligen Rechteinhabers einzuholen.

Weitere Details zur Lizenz entnehmen Sie bitte der Lizenzinformation auf http://creativecommons.org/licenses/by/4.0/deed.de.

\section{Literatur}

DIN 45 645-2 (2012) Ermittlung von Beurteilungspegeln aus Messungen, Teil 2: Ermittlung des Beurteilungspegels am Arbeitsplatz bei Tätigkeiten unterhalb des Pegelbereiches der Gehörgefährdung. Beuth Verlag Berlin

Fastl H, Zwicker E (2007) Psychoacustics, facts and models, 3. Aufl. Springer, Berlin

Hesse JM, Irle H, Strasser H (1994) Laborexperimentelle Untersuchungen zur Gehörschädlichkeit von Impulsschall. Z Arb Wiss 48(4):237-244

Hoffmann H, v. Lüpke A (1979) Null Dezibel + Null Dezibel = Drei Dezibel. Schmidt, Berlin

Irle $\mathrm{H}$, Strasser H (2005) Methods for quantifying hearing threshold shifts of sound exposures and for depicting the parameters $\mathrm{TTS}_{2}, \mathrm{t}(0 \mathrm{~dB})$, and IRTTS indicating the physiological costs to the hearing. In: Strasser H (Hrsg) Traditional rating of noise versus physiological costs of sound exposures to the hearing. IOS Press, Amsterdam, Berlin Oxford, Tokyo, Washington, DC, S 53-66 (Chapter 4)

Irle H, Hesse JM, Strasser H (1998) Physiological cost of energy-equivalent noise exposures with a rating level of $85 \mathrm{~dB}(\mathrm{~A})$ - hearing threshold shifts associated with energetically negligible continuous and impulse noise. Int J Ind Ergon 21:451-463

Irle H, Körner F, Strasser H (2005) Vertäubung und Erholung des Gehörs nach energie-äquivalenter Beschallung durch Heavy-Metal, Techno und Klassische Musik. Z Lärmbekämpfung 52:46-54

Irle H, Hesse JM, Strasser H (2006) Physiological costs of noise exposures: temporary threshold shifts. In: Karwowski W (Hrsg) International encyclopedia of ergonomics and human factors. Taylor \& Francis, London, New York, S 1846-1853

Miller JD (1974) Effects of noise on people. J Acoust Soc Am 56:729-764

N. N. (2007) Verordnung zum Schutz der Beschäftigten vor Gefährdungen durch Lärm und Vibrationen (Lärm- und Vibrations-Arbeitsschutzverordnung - LärmVibrationsArb.SchV.) vom 6. März 2007. BGBI., S 261, zul. geänd. durch Art. 5 der Verordnung vom 18. Dezember 2008, BGBI. I, S 2768

Strasser H (Hrsg) (2005) Traditional rating of noise versus physiological costs of sound exposures to the hearing. IOS Press, Amsterdam, Berlin, Oxford, Tokyo, Washington, DC, S 227

Strasser H (2011) Dosis-Maxime und Energie-Äquivalenz bei der Beurteilung von Lärm sowie physiologischen Kosten von Schallbelastungen. Lärmbekämpfung 6(2):51-58

Strasser H (2017) Trends in der Messung, Bewertung und Beurteilung von Lärm sowie seinen auralen und extra-auralen Wirkungen Ein Positionspapier. Z Arb Wiss 71(3):189-203

Strasser H, Irle H (2006) Noise: measuring, evaluation, and rating in ergonomics. In: Karwowski W (Hrsg) International encyclopedia of ergonomics and human factors. Taylor \& Francis, London, S 44-851

Strasser H, Irle H, Scholz R (2005) Physiologische Kosten energieäquivalenter akustischer Belastungen durch „Weißes Rauschen“, Industrielärm, Heavy Metal-Musik und Klassische Musik. Z Arb Wiss 59:395-407

Strasser H, Chiu M-C, Irle H, Grünig T (2007) Threshold shifts and restitution of the hearing after energy-equivalent narrow-band and wide-band noise exposures. Proceedings-CD Internoise 2007, Istanbul, S 1-10

Strasser H, Chiu M-C, Müller O (2008a) Threshold shifts and restitution of the hearing after energy-equivalent noise exposures with an equal NRC-value and non-equal frequency composition. Proceedings-CD of 9th International Congress on Noise as a Public Health Problem (ICBEN) Foxwoods, Connecticut, S 1-9

Strasser H, Chiu M-C, Irle H, Wagener A (2008b) Threshold shifts and restitution of the hearing after different music exposures. Theor Issues Ergonomics Sci 9(5):405-424

Strasser H, Wang M-J, Chiu M-C, Müller O (2009) Physiological costs and hearing risks associated with Chinese classical music and energy-equivalent sound exposures with differing frequency composition. Proceedings of the 17th Triennial Congress of the International Ergonomics Association, Beijing, S 1-9

VDI 2058 Blatt 3 (2014) Beurteilung von Lärm am Arbeitsplatz unter Berücksichtigung unterschiedlicher Tätigkeiten. Beuth Verlag, Berlin 